

\title{
Remarks on Affine Variations on the Ellipsoid
}

\author{
Martin Wiehe*
}

\begin{abstract}
We extend a variational result of Blaschke and give some further stability results.
\end{abstract}

MS-Classification: 53A15, 33C35.

\section{Introduction}

As a reference for affine differential geometry we use [SSV91] from which we adopt most of the notations.

Let $A^{n+1}$ be an $(n+1)$-dimensional real affine space and $M$ an $n$-dimensional $(n \geq 2)$ connected and orientable manifold. Let $f: M \longrightarrow A^{n+1}$ be a $C^{\infty}$-immersion. Suppose that the Euclidean second fundamental form is nondegenerate on $f$ - which means that $f$ is regular - then the equiaffine conormal (Blaschke conormal) is given by $Y:=\left|H_{n}(E)\right|^{-\frac{1}{n+2}} \mu$ where $H_{n}(E)$ is the Euclidean Gauß-Kronecker curvature and $\mu$ the Euclidean normal. The equiaffine normal (Blaschke normal) is implicitly defined by

$$
<Y, y>=1 \quad \text { and } \quad<d Y, y>=0 .
$$

The structure equations have the following form:

$$
\begin{aligned}
\bar{\nabla}_{u} d f(v) & =d f\left(\nabla_{u} v\right)+h(u, v) y \\
d y(v) & =-d f(S v)
\end{aligned}
$$

for all $u, v \in \mathfrak{X}(M)$. With $\mathfrak{X}(M)$ we denote the $C^{\infty}$-module of vector fields over $M$ and with $\bar{\nabla}$ the standard flat connection on $A^{n+1}$.

$\nabla$ is a torsion free affine connection, $h$ a symmetric $(0,2)$-tensor field, $S$ a $(1,1)$-tensor field. In the case of a regular hypersurface, $h$ is a semi Riemannian metric, hence we do all metric operations (also such in standard local notation) with respect to $h$; e.g. $\triangle g=\operatorname{trace}_{h}\{\operatorname{Hess}(h) g\}=$ trace $_{h}\{\nabla(h) \nabla(h) g\}$ for $g \in C^{\infty}(M)$ and $\nabla(h)$ the Levi-Civita connection of $h$.

Recall that the cubic tensor $C:=\nabla-\nabla(h)$ is traceless (apolarity).

The affine mean curvature $H$ is defined as $n H=\operatorname{trace} S$. If $f$ is an ovaloid ( $M$ is compact without boundary) it is well known that the ellipsoid is characterized by $H=$ constant.

\footnotetext{
*Supported by DFG (Graduiertenkolleg HU-TU Berlin)
} 
Definition 1.1. Let $I:=(-\varepsilon, \varepsilon)$ for some $\varepsilon>0$ and $f^{t}: M \times I \rightarrow A^{n+1}$ be a $C^{\infty}$ one parameter family of regular hypersurfaces. If $f^{0}=f$ and $f^{t}-f$ is of compact support then $f^{t}$ is called a variation of $f$.

Define the function $\alpha^{t}$ and the vector field $\beta^{t}$ for $t \in I$ by $f^{t}=: f+d f\left(\beta^{t}\right)+\alpha^{t} y$. With $(\cdot)^{\prime}:=$ $\left.\frac{\partial}{\partial t}(\cdot)\right|_{t=0}$ set $\phi(p):=\alpha(p)^{\prime}$ and $\psi^{i}:=\beta^{i \prime}, \psi=\psi^{i} \partial_{i}$. A variation is called normal iff $d f^{\prime}(u)$ is tangential for every $u \in \mathfrak{X}(M)$ and linear iff $f^{\prime}=0$. In terms of the equiaffine representation $\{\phi, \psi\}$ of the infinitesimal variation $f^{\prime}$ the normality condition is equivalent to $\psi=-\operatorname{grad}_{h} \phi$.

For the equiaffine area $A(M):=\int_{M} \omega(h)$ it is well known (see [Ca182] or [NS94, III.11]) that:

$$
A(M)^{\prime}=-\frac{n(n+1)}{n+2} \int_{M} H \phi \omega(h)
$$

for all variations $f^{t}$. This follows easily from

$$
\begin{aligned}
\omega(h)^{\prime} & =\frac{1}{n+2}[\Delta \phi-n(n+1) H \phi+(n+2) \operatorname{div}(\psi)] \omega(h) \\
S_{i}^{j \prime} & =\phi S_{i}^{r} S_{r}^{j}+g S_{i}^{j}-\left(\nabla_{\partial_{r}} \psi\right)^{j} S_{i}^{r}+\left(\nabla_{\partial_{i}}\left(\operatorname{grad}_{h} g+S \operatorname{grad}_{h} \phi+S \psi\right)\right)^{j}
\end{aligned}
$$

with $g:=\frac{1}{n+2}(\triangle \phi+n H \phi)$. For (1.1) see [NS94, III.11], and (1.2) could be obtained following the calculations for (4.20) in [Cal82].

\section{Some stability results}

We start with a statement based on an observation of U. Simon.

Lemma 2.1. Let $B: X \rightarrow X$ be a linear operator on a real linear space $X$ and $r \in \mathbb{N}$. Suppose that $\left\{\lambda_{1}, \ldots, \lambda_{r}\right\} \subset \mathbb{R}$ with $\lambda_{i} \neq \lambda_{j}$ and there exists $\beta \in V \backslash\{0\}$ such that

$$
\left(B-\lambda_{1} i d\right) \cdots\left(B-\lambda_{r} i d\right) \stackrel{*}{\beta}=0 .
$$

Then there exists a nonempty set $\mathcal{A} \subset\{1, \ldots, r\}$ such that $\lambda_{i}$ are eigenvalues of $B$ for all $i \in \mathcal{A}$ and $\beta=\sum_{i \in \mathcal{A}} p_{i}$ where $p_{i} \in E_{\lambda_{i}}$ and $E_{\lambda_{i}}$ denotes the eigenspace of $\lambda_{i}$.

Proof. (Sketch). The statement is proved by induction on $r$. The main steps are: Define $\beta_{1}=$ $\left(B-\lambda_{1} i d\right) \beta$ and $\beta_{2}=\left(B-\lambda_{2} i d\right) \beta$. Hence $\Pi_{j \neq i}\left(B-\lambda_{j} i d\right) \beta_{i}=0$ for $i \in\{1,2\}$. The lemma finally follows from $\beta=\frac{\beta_{1}-\beta_{2}}{\lambda_{2}-\lambda_{1}}$.

Let $T$ be an affine invariant induced by the regular hypersurface $f$. Then $f$ is called $T$-equiaffinestable iff $A(M)^{\prime \prime} \geq 0$ holds for every variation satisfying $T^{\prime}=0$.

For an ellipsoid with equiaffine normal the eigenvalues of the Laplacian are $\lambda_{i}=-i(n+i-1) H$ for $i \in \mathbb{N} \cup\{0\}$. The eigenfunctions corresponding to $i=1,2, \ldots$ are called first, second, ... order spherical harmonics.

Theorem 2.2. For the class of linear normal variations the ellipsoid is H-equiaffine-stable. 
Proof. Let $f: M \rightarrow A^{n+1}$ be an ellipsoid endowed with the equiaffine normal. Hence $H=$ constant $\neq 0$ which implies $S=H i d$. The trace of (1.2) reduces to

$$
H^{\prime}=\frac{1}{n(n+2)}(\triangle-\lambda)(\triangle-\mu) \phi
$$

with $\lambda=-n H$ and $\mu=-2 H(n+1)$. Since the deformation of $f$ satisfies $H^{\prime}=0$ we obtain $\phi=p_{\lambda}+p_{\mu}$ where $p_{\lambda} \in E_{\lambda}$ and $p_{\mu} \in E_{\mu}$. Using (1.1) for normal variations we obtain

$$
\begin{aligned}
-\frac{n+2}{n(n+1)} A(M)^{\prime \prime} & =\left(\int_{M} H \phi \omega(h)\right)^{\prime}=\int_{M} H \phi \omega(h)^{\prime}=\frac{n+1}{n+2} H \int_{M} \phi[\Delta \phi+n H \phi] \omega(h) \\
& =\frac{(n+1)(n-1)}{n+2} H^{2} \int_{M}\left(p_{\lambda}+p_{\mu}\right) p_{\mu}=\frac{(n+1)(n-1)}{n+2} H^{2} \int_{M} p_{\mu}^{2} \omega(h) \geq 0 .
\end{aligned}
$$

Corollary 2.3. For the class of linear normal variations the ellipsoid is $k$-equiaffine-stable, where $k$ is the normed scalar curvature of $h$.

Proof. It is well known that $k=J+H$ where $n(n-1) J=h(C, C)$ is the Pick invariant. Since $C=0$ characterizes the ellipsoid we get $k^{\prime}=H^{\prime}$.

\section{Trivial variations}

The following definition is only meaningful for compact hypersurfaces without boundary, but we formulate it in general. Our definition of "trivial variations" is appropriate to understand why second order spherical harmonics appear in 2.2 (see the proof). Other definitions of trivial variations, e.g. given in [PS87], do not fit in this way.

Definition 3.1. Let $f$ be a regular hypersurface with equiaffine normal $y$. A variation $f^{t}$ of $f$ is called equiaffine-trivial iff there exists a one parameter family of diffeomorphisms $\theta^{t}: M \rightarrow M$ such that $\theta^{0}=i d$ and $h^{t}=\theta^{t *} h, \nabla^{t}=\theta^{t *} \nabla$. Here $\theta^{t *}$ denotes the pullback via $\theta^{t}$.

Remark 3.2 From the uniqueness theorem it follows that the variation $f^{t}$ of $f$ is trivial iff there exist one parameter families $A^{t} \in S L(n+1, \mathbb{R})$ and $b^{t} \in \mathbb{R}^{n+1}$ with $A^{0}=i d$ and $b^{0}=0$ such that $f^{t}=A^{t}\left(f \circ \theta^{t}\right)+b^{t}$.

An easy consequence of (2.3) is

Proposition 3.3. Let $f$ be an ellipsoid endowed with the equiaffine normal. If an equiaffine infinitesimal variation $\phi$ of $f$ is equiaffine-trivial then $\phi=p_{2}+p_{1}$ where $p_{2} \in E_{2}$ and $p_{1} \in E_{1}$.

Lemma 3.4. Let $f$ be an ellipsoid endowed with the equiaffine normal. Then

(i) $p_{1}$ is a first order spherical harmonic iff there exists $\mathfrak{b} \in V^{n+1}$ such that $p_{1}=\langle Y, \mathfrak{b}\rangle$.

(ii) $p_{2}$ is a second order spherical harmonic iff there exists $\mathfrak{A} \in L(n+1, \mathbb{R})$ with trace $A=0$ such that $p_{2}=\langle Y, \mathfrak{A} y\rangle$. 
Proof. (i) is a special case of Satz 3.1 in [Sch67] and (ii) is a conclusion of Satz 3.5 in [Led95].

Now we can prove

Theorem 3.5. Let $f$ be an ellipsoid endowed with the equiaffine normal. Then a normal infinitesimal variation $\phi$ of $f$ is equiaffine-trivial iff $\phi=p_{2}+p_{1}$ where $p_{2} \in E_{2}$ and $p_{1} \in E_{1}$.

Proof. Assume that $\phi=p_{2}+p_{1}$. Define $b^{t}=t \mathfrak{b}$ with $\mathfrak{b}$ given from 3.4(i). Define a one parameter family $L^{t}:=\mathfrak{A}$ with $\mathfrak{A}$ given from 3.4(ii). Since trace $\mathfrak{A}=0$ there exists a one parameter family $A^{t} \in S l(n+1, \mathbb{R})$ with $A^{\prime}=\mathfrak{A}$ and $A^{0}=i d$. From Proposition 4.1 in [Cal82] we get the existence of a family of diffeomorphisms $\theta^{t}$ such that $f^{t}:=A^{t} f \circ \theta^{t}+b^{t}$ is a normal variation.

Hence we get a generalization of a result in [B1a23, $\S 90]$.

Corollary 3.6. Let $f$ be an ellipsoid endowed with the equiaffine normal then any normal infinitesimal variation with

(i) $H^{\prime}=0$ is equiaffine-trivial;

(ii) $k^{\prime}=0$ is equiaffine-trivial.

A similar result was obtained in [Lei98] considering restricted trivial variations with $\theta^{t}=i d$.

\section{References}

[Bla23] W. Blaschke, Vorlesungen über Differentialgeometrie I. Affine Differentialgeometrie, Springer Berlin, 1923.

[Cal82] E. Calabi, Hypersurfaces with maximal affinely invariant area, Am. J. Math. 104 (1982), 91-126.

[Led95] J. Leder, Erzeugung kubischer Formen durch Funktionen, Diploma thesis, TU-Berlin, 1995.

[Lei98] K. Leichtweiß, On the infinitesimal affine rigidity of ellipsoids in the $n$-space, Tech. report, $\mathrm{U}$ Stuttgart, 1998.

[Li88] A. M. Li, Variational formulas for higher affine mean curvatures, Result. Math. 13 (1988), 318326.

[NS94] K. Nomizu and T. Sasaki, Affine Differential Geometry, Cambridge University Press, 1994.

[PS87] G. Penn and U. Simon, Deformations of hypersurfaces in equiaffine differential geometry, Ann. Global Anal. Geom. 5, No. 2 (1987), 123-131.

[Sch67] R. Schneider, Zur affinen Differentialgeometrie im Großen. I, Math. Z. 101 (1967), 375-406.

[SSV91] U. Simon, A. Schwenk-Schellschmidt, and H. Viesel, Introduction to the Affine Differential Geometry of Hypersurfaces, Science University of Tokyo, 1991, Lecture Notes (ISBN 37983 15299).

[Vos91] K. Voss, Variation of curvature integrals, Result. Math. 20 (1991), 789-796.

Address: Martin Wiehe, TU Berlin, FB Mathematik, MA 8-3,

Strasse des 17. Juni 135, D-10623 Berlin.

wiehe@math.tu-berlin.de 